\title{
THE PLANETARY SYSTEM TO KIC 11442793: A COMPACT ANALOGUE TO THE SOLAR SYSTEM
}

\author{
J. Cabrera ${ }^{1}$, Sz. Csizmadia ${ }^{1}$, H. Lehmann ${ }^{2}$, R. Dvorak ${ }^{3}$, D. Gandolfi ${ }^{4,5}$, H. Rauer ${ }^{1,6}$, \\ A. Erikson ${ }^{1}$, C. Dreyer ${ }^{1}$, Ph. EIgmüller ${ }^{1}$, AND A. Hatzes ${ }^{2}$ \\ ${ }^{1}$ Institute of Planetary Research, German Aerospace Center, Rutherfordstrasse 2, D-12489 Berlin, Germany \\ ${ }^{2}$ Thüringer Landessternwarte, D-07778 Tautenburg, Germany \\ ${ }^{3}$ Universitätssternwarte Wien, Türkenschanzstr. 17, A-1180 Wien, Austria \\ ${ }^{4}$ Research and Scientific Support Department, ESTEC/ESA, P.O. Box 299, 2200 AG Noordwijk, The Netherlands \\ ${ }^{5}$ INAF-Catania Astrophysical Observatory, Via S. Sofia 78, I-95123 Catania, Italy \\ ${ }^{6}$ Center for Astronomy and Astrophysics, TU Berlin, Hardenbergstr. 36, D-10623 Berlin, Germany \\ Received 2013 July 9; accepted 2013 November 8; published 2013 December 31
}

\begin{abstract}
We announce the discovery of a planetary system with seven transiting planets around a Kepler target, a current record for transiting systems. Planets $\mathrm{b}, \mathrm{c}, \mathrm{e}$, and $\mathrm{f}$ are reported for the first time in this work. Planets $\mathrm{d}$, g, and $\mathrm{h}$ were previously reported in the literature, although here we revise their orbital parameters and validate their planetary nature. Planets $\mathrm{h}$ and $\mathrm{g}$ are gas giants and show strong dynamical interactions. The orbit of planet $\mathrm{g}$ is perturbed in such a way that its orbital period changes by $25.7 \mathrm{hr}$ between two consecutive transits during the length of the observations, which is the largest such perturbation found so far. The rest of the planets also show mutual interactions: planets $\mathrm{d}, \mathrm{e}$, and $\mathrm{f}$ are super-Earths close to a mean motion resonance chain (2:3:4), and planets $\mathrm{b}$ and c, with sizes below 2 Earth radii, are within $0.5 \%$ of the $4: 5$ mean motion resonance. This complex system presents some similarities to our solar system, with small planets in inner orbits and gas giants in outer orbits. It is, however, more compact. The outer planet has an orbital distance around $1 \mathrm{AU}$, and the relative position of the gas giants is opposite to that of Jupiter and Saturn, which is closer to the expected result of planet formation theories. The dynamical interactions between planets are also much richer.
\end{abstract}

Key words: planetary systems - planets and satellites: detection - planets and satellites: dynamical evolution and stability - stars: individual (KIC 11442793, KOI 351, Kepler-90) - techniques: photometric techniques: spectroscopic

Online-only material: color figures

\section{INTRODUCTION}

Finding planetary systems similar to our own is one of the main goals of exoplanet searches. It is of particular interest if such systems show planetary transits, since multiple transiting planetary systems provide crucial information for the understanding of planet formation and evolution (Ford \& Gaudi 2006). Mutual dynamical interactions between planets especially require an additional effort to understand their origin and to justify their long-term stability. Unfortunately, such systems are difficult to find because of the low geometrical probability for transiting planets. The satellite Kepler (Borucki et al. 2010) has observed the planetary system orbiting the star KIC 11442793 almost continuously for more than four years. The Kepler team has published the parameters of three transiting candidates around this star (Batalha et al. 2013) with the identification numbers KOI 351.01, .02, and .03. A careful analysis of the light curve with the transit detection algorithm Détection Spécialisée de Transits (DST) (Cabrera et al. 2012) reveals the presence of four additional transiting planets, making this system the most populated among the transiting ones. These four planets are reported here for the first time (see the results of Ofir \& Dreizler 2013; Huang et al. 2013; Tenenbaum et al. 20137). Considering the magnitude of the star (13.7 mag in SDSS $r$ ) and the characteristics of the transiting candidates, we were not able to independently confirm the planets by measuring their masses with radial velocity. However, we have performed the

\footnotetext{
7 While this paper was in referee process, Schmitt et al. submitted a paper to The Astronomical Journal with an independent characterization of this system.
}

following steps to validate the planetary nature of the candidates: (1) medium resolution spectra of the star were taken with the Coudé-Echelle spectrograph at the Tautenburg observatory, characterizing the host star as a solar-like dwarf; (2) the analysis of the Kepler photometry, including the study of the motion of the point-spread function centroid (Batalha et al. 2010), which does not reveal any hint of the presence of a contaminating eclipsing binary; (3) the analysis of the timing of the eclipses reveals that the planetary candidates are dynamically interacting with each other; and finally, (4) a stability analysis of the system with the orbital dynamics integrator Mercury (Chambers 1999) reveals that, for the system to be stable, all of the planetary candidates must have planetary masses. Therefore, in this paper, we validate the planetary nature of the seven candidates.

\section{STELLAR CHARACTERIZATION}

In order to characterize the host star, five spectra were taken on 2013 June 6 and 7, with the Coudé-Echelle spectrograph attached to the $2 \mathrm{~m}$ telescope at the Thüringer Landessternwarte Tautenburg. The wavelength coverage was $472-736 \mathrm{~nm}$, and a 2 arcsec slit provided a spectral resolving power of 32,000 . The exposure time for each spectrum was 40 minutes.

The spectra were reduced using standard ESO-MIDAS packages. The reduction steps included filtering of cosmic rays, background, and straylight subtraction, flat fielding using a halogen lamp, optimum extraction of diffraction orders, and wavelength calibration using a ThAr lamp. Due to the low signal-to-noise ratio $(\mathrm{S} / \mathrm{N})$ of a single spectrum, it was difficult to define the local continuum. Because no radial velocity shifts between the 


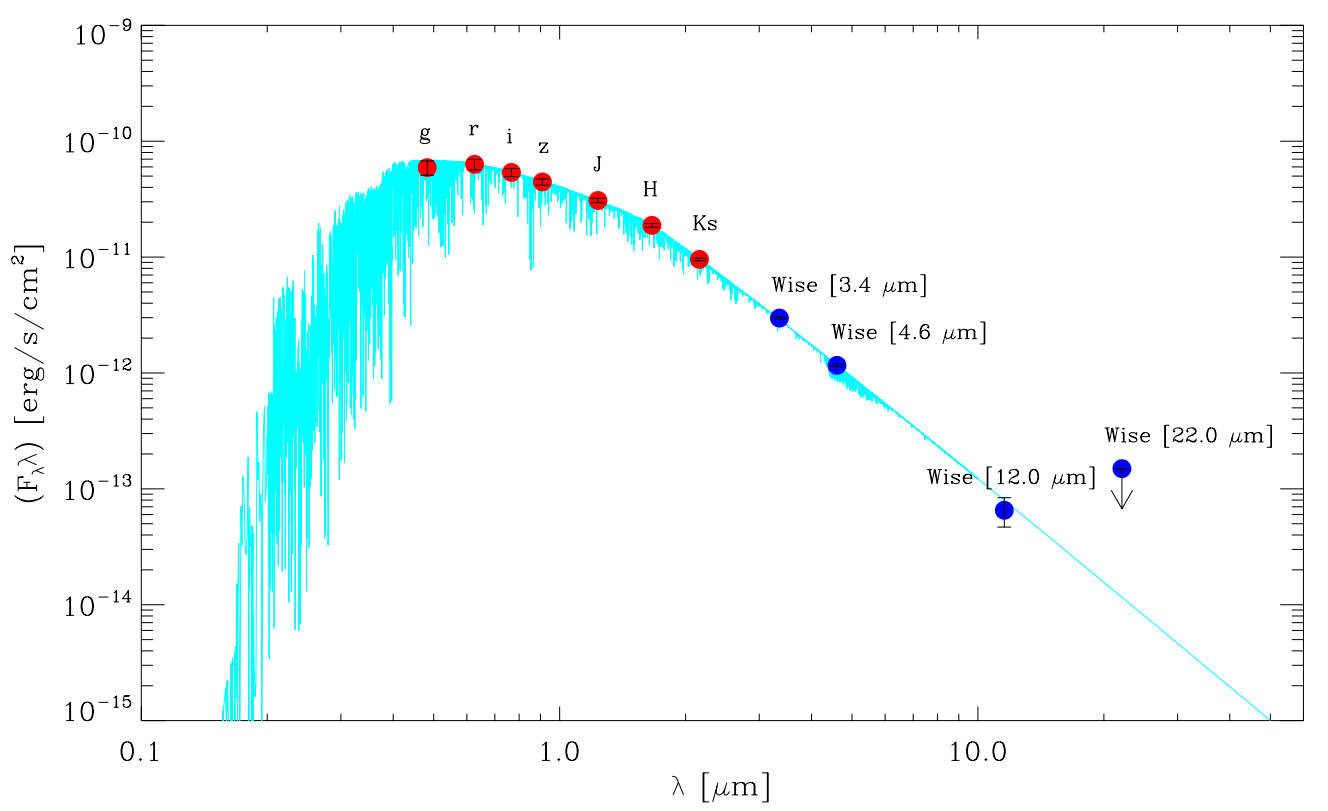

Figure 1. Dereddened spectral energy distribution of KIC 11442793. The optical SDSS- $g,-r,-i,-z$ photometry is from the Kepler Input Catalog. Infrared $J, H, K s$ and $W 1, W 2, W 3, W 4$ data are taken from the 2MASS (Cutri et al. 2003) and WISE (Wright et al. 2010) databases, respectively. The NextGen model spectrum by Hauschildt et al. (1999) with the same photospheric parameters as KIC 11442793 and scaled to the stellar radius and distance is overplotted with a light blue line.

(A color version of this figure is available in the online journal.)

Table 1

Derived Atmospheric Parameters for the Star

\begin{tabular}{lcc}
\hline \hline & Full Grid & $\log g$ Fixed \\
\hline$T_{\text {eff }}(\mathrm{K})$ & $5930 \pm 320$ & $6080_{-170}^{+260}$ \\
$\log g(\mathrm{cgs})$ & $4.0 \pm 0.5$ & 4.4 (fixed) \\
$v_{\text {mic }}\left(\mathrm{km} \mathrm{s}^{-1}\right)$ & $1.2 \pm 0.6$ & $1.2 \pm 0.6$ \\
$(\mathrm{M} / \mathrm{H})\left(\mathrm{dex}^{-1}\right.$ & $-0.17 \pm 0.21$ & $-0.12 \pm 0.18$ \\
$v \sin i\left(\mathrm{~km} \mathrm{~s}^{-1}\right)$ & $4.6 \pm 2.1$ & $4.6 \pm 2.1$ \\
\hline
\end{tabular}

single spectra could be found, we repeated the reduction using the co-added raw spectra. The continuum of the resulting mean spectrum was then well enough defined for a proper normalization. The $\mathrm{S} / \mathrm{N}$ of the mean spectrum, measured from some almost line-free parts of the continuum, was about 19.

We used the spectral synthesis method, which compared the observed spectrum with synthetic spectra computed on a grid in atmospheric parameters. The synthetic spectra were computed with the SynthV program (Tsymbal 1996), based on a library of atmosphere models calculated with the LLmodels code (Shulyak et al. 2004). The error estimation was done from $\chi^{2}$ statistics taking all interdependencies between the different parameters into account (Lehmann et al. 2011).

The step widths of the grid were $100 \mathrm{~K}$ in $T_{\text {eff }}, 0.1$ dex in $\log g$, 0.1 dex in $[\mathrm{M} / \mathrm{H}], 0.5 \mathrm{~km} \mathrm{~s}^{-1}$ in microturbulent velocity, and $1 \mathrm{~km} \mathrm{~s}^{-1}$ in $v \sin i$; where $[\mathrm{M} / \mathrm{H}]$ means scaled solar abundances. For the determination of $v \sin i$, we used the metal line-rich wavelengths region, 491-567 nm. For all other parameters, the wavelength range utilized was $472-567 \mathrm{~nm}$ which also includes $H_{\beta}$.

Table 1 lists the results obtained from the full grid in all parameters. The large uncertainties mainly originate from the large ambiguities between the different parameters and from the low S/N of the observed spectrum. We use a compilation of empirical values of stellar parameters from Gray (2005). Comparing our results from the full grid search with the literature data, we see that we can exclude luminosity class III stars because of the values of $\log g$ and $\operatorname{vsin} i$. The $T_{\text {eff }}$ derived from spectral analysis lies within the uncertainties between 5600 and $6250 \mathrm{~K}$ which is consistent with dwarfs of spectral type G6 to F6. Based on the measured $\log g$, we cannot determine if the star is slightly evolved. Assuming that the star is a typical main sequence star of early G-type, we can adopt a $\log g$ of 4.4, which lies within the measurement error, to obtain a better constraint on $T_{\text {eff }}$ and a slightly higher value for the metallicity (last column of Table 1). Under this assumption, we obtain $T_{\text {eff }}$ between 5910 and $6340 \mathrm{~K}$, corresponding to spectral types between G1 and F6. The corresponding ranges in mass and radius are relatively small, between 1.1 and $1.3 M_{\text {sun }}$ and 1.1 and $1.3 R_{\text {sun }}$.

\subsection{Reddening and Distance}

We determined the interstellar extinction, $A_{\mathrm{v}}$, and distance, $d$, to KIC 11442793 by applying the method described in Gandolfi et al. (2008). This technique is based on the simultaneous fit of the observed stellar colors with theoretical magnitudes obtained from the NextGen model spectrum (Hauschildt et al. 1999) with the same photospheric parameters as the target star. For KIC 1144279,3 we used SDSS, 2MASS, and WISE photometry (see Table 2 and Figure 1). We excluded the $W_{3}$ and $W_{4}$ WISE magnitudes, as the former has a $\mathrm{S} / \mathrm{N}$ of 3.5 and the latter is only an upper limit. Assuming a normal extinction $\left(R_{\mathrm{v}}=3.1\right)$ and a black body emission at the stellar effective temperature and radius, we found that the star reddening amounts to $A_{\mathrm{v}}=0.15 \pm 0.10 \mathrm{mag}$ and that the distance to KIC 11442793 is $d=780 \pm 100 \mathrm{pc}$.

\section{LIGHT CURVE ANALYSIS}

Kepler observations of KIC 11442793 extend for 1340 days with a duty cycle of $82 \%$. The light curve, shown in Figure 2, reveals that the host star is not particularly active. It barely shows hints of some variations compatible with the evolution of stellar spots on its surface, with an amplitude of $0.1 \%$

We have applied a detrending algorithm to treat the stellar activity optimized for the CoRoT mission (Baglin et al. 2006), 


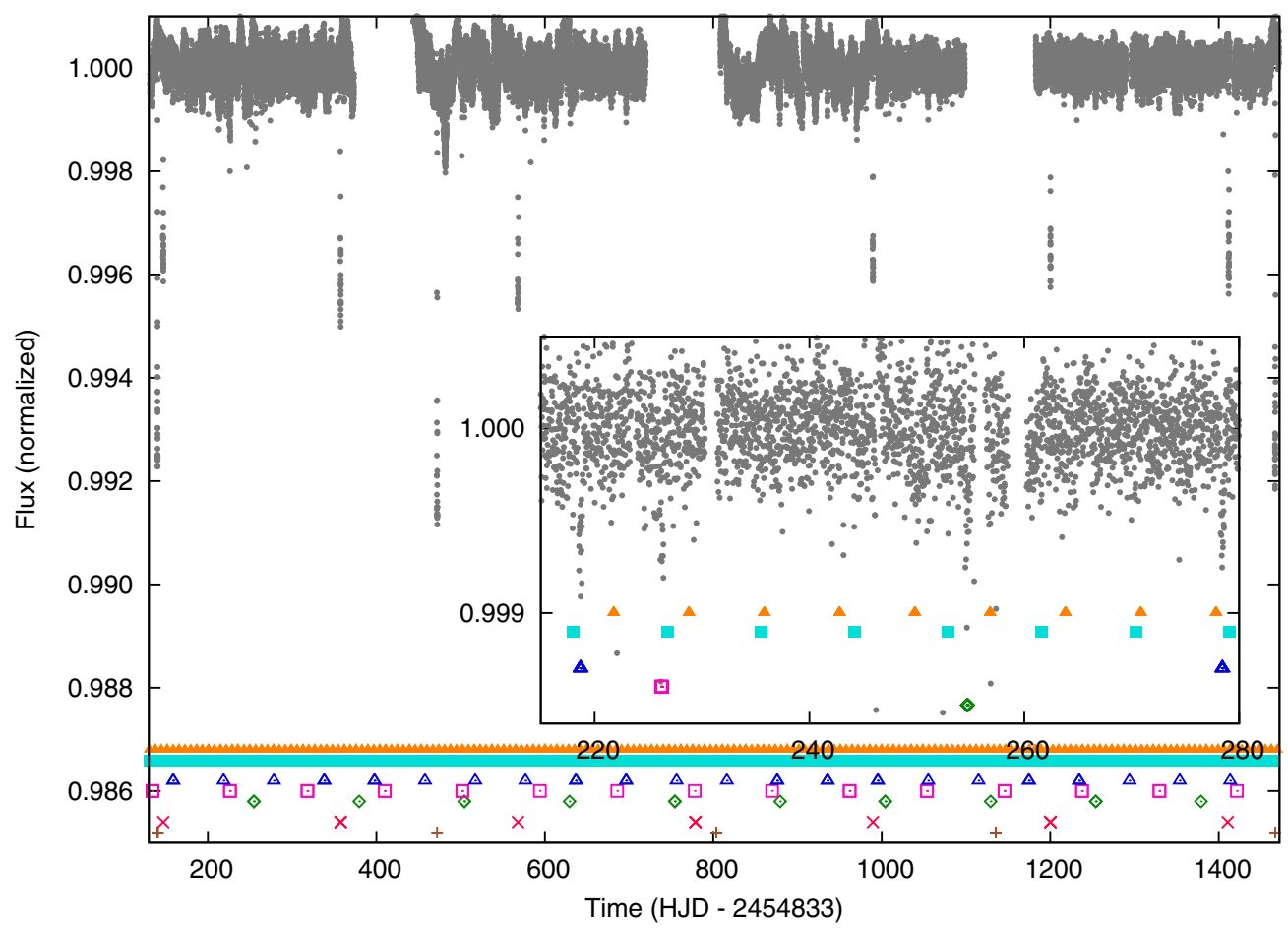

Figure 2. Public raw light curve of KIC 11442793. The seven sets of periodic transits are indicated with symbols of different colors: planet h with red plus signs, planet $\mathrm{g}$ with red crosses, planet $\mathrm{f}$ with green diamonds, planet e with magenta squares, planet $\mathrm{d}$ with blue triangles, planet $\mathrm{c}$ with turquoise filled squares, and planet $\mathrm{b}$ with orange filled triangles. In the enlarged region the stellar variability has been subtracted to show a subset of the shallower transits.

(A color version of this figure is available in the online journal.)

Table 2

Kepler, GSC2.3, USNO-A2, and 2MASS Identifiers of the Target Star

\begin{tabular}{lc}
\hline \hline Main identifiers & \\
\hline Kepler IDs & KIC 11442793, KOI 351, Kepler-90 \\
GSC2.3 ID & N2EM001018 \\
USNO-A2 ID & $1350-10067455$ \\
2MASS ID & $18574403+4918185$ \\
\hline
\end{tabular}

\begin{tabular}{|c|c|c|}
\hline \multicolumn{3}{|l|}{ Equatorial coordinates } \\
\hline R.A. $(\mathrm{J} 2000) 18^{\mathrm{h}} 57^{\mathrm{m}} 44^{\mathrm{s}} .038$ & decl. $(\mathrm{J} 2000)+49^{\circ} 18^{\prime} 18^{\prime \prime} .58$ & \\
\hline \multicolumn{3}{|l|}{ Magnitudes } \\
\hline Filter $\left(\lambda_{\text {eff }}\right)$ & Mag & Uncertainty \\
\hline$g \quad(0.48 \mu \mathrm{m})$ & 14.139 & 0.030 \\
\hline$r \quad(0.63 \mu \mathrm{m})$ & 13.741 & 0.030 \\
\hline$i \quad(0.77 \mu \mathrm{m})$ & 13.660 & 0.030 \\
\hline$z \quad(0.91 \mu \mathrm{m})$ & 13.634 & 0.030 \\
\hline$J \quad(1.24 \mu \mathrm{m})$ & 12.790 & 0.029 \\
\hline$H \quad(1.66 \mu \mathrm{m})$ & 12.531 & 0.033 \\
\hline$K s(2.16 \mu \mathrm{m})$ & 12.482 & 0.024 \\
\hline$W_{1}(3.35 \mu \mathrm{m})$ & 12.429 & 0.024 \\
\hline$W_{2}(4.60 \mu \mathrm{m})$ & 12.462 & 0.024 \\
\hline$W_{3}(11.56 \mu \mathrm{m})$ & 12.750 & 0.308 \\
\hline$W_{4}(22.09 \mu \mathrm{m})$ & $9.702^{\mathrm{a}}$ & $\ldots$ \\
\hline
\end{tabular}

Notes. Equatorial coordinates and optical SDSS- $g,-r,-i,-z$ photometry are from the Kepler Input Catalogue. Infrared $J, H, K s$ and $W 1, W 2, W 3, W 4$ data are taken from the 2MASS (Cutri et al. 2003) and WISE (Wright et al. 2010) databases, respectively.

${ }^{\text {a }}$ Upper limit.

but adapted to the treatment of Kepler data (Cabrera et al. 2012). Then we have applied the transit detection algorithm DST (Cabrera et al. 2012) to search for the periodic signature of transiting planets.
We confirm the detection of the candidates KOI 351.01, .02, and .03, previously announced (Batalha et al. 2013), and we assign them the identifications KIC $11442793 \mathrm{~h}, \mathrm{~g}$, and d. We present the discovery of four additional candidates, b, c, e, and $f$, reported for the first time here. The ephemerides of these objects are given in Table 3. The orbital ephemerides have been calculated as follows: the transit detection algorithm DST provides preliminary values of the period, epoch, depth, and duration of the transiting candidates. With this information, we first separately fit the transits of every candidate. Then, we make a weighted linear fit to the epochs of the individual transits; the slope of the fit is the period and the intercept is the epoch. The residuals between the linear fit and the actual position of the transits (observed minus calculated, $O-C$ ) are usually referred to as transit timing variations (TTVs), which are discussed later in Section 5.

\section{PLANETARY PARAMETER MODELING}

Several planets in this system show significant TTVs, described in Section 5, which need to be removed before proceeding with the modeling of the planetary parameters. We use an iterative method to correct for this effect, similar to the one described by Alapini \& Aigrain (2009), but accounting for the TTVs. We take a geometrical model of the transit based on the preliminary value of the planetary parameters obtained by the detection method. We use a genetic algorithm (Geem et al. 2001) to fit the value of the epoch, fixing the other transit parameters. For every trial value of the epoch, we correct for stellar activity in a region covering 10 times the transit duration with a second order Legendre polynomial (first order for planets $b$ and c). The polynomial is interpolated in the expected region of the transit to preserve the transit shape. We then fold the light 
Table 3

Planetary Parameters

\begin{tabular}{|c|c|c|c|}
\hline KIC 11442793 h (KOI 351.01) & \multicolumn{3}{|c|}{ KIC 11442793 d (KOI 351.03) } \\
\hline Period (days) & $331.60059 \pm 0.00037$ & Period (days) & $59.73667 \pm 0.00038$ \\
\hline Epoch (HJD-2454833) & $140.49631 \pm 0.00082$ & Epoch (HJD-2454833) & $158.9656 \pm 0.0042$ \\
\hline Duration (hr) & $14.737 \pm 0.046$ & Duration (hr) & $8.40 \pm 0.19$ \\
\hline$a / R_{s}$ & $180.7 \pm 4.7$ & $a / R_{s}$ & $56.1 \pm 4.8$ \\
\hline$a(\mathrm{AU})$ & $1.01 \pm 0.11$ & $a(\mathrm{AU})$ & $0.32 \pm 0.05$ \\
\hline$R_{p} / R_{s}$ & $0.0866 \pm 0.0007$ & $R_{p} / R_{s}$ & $0.0219 \pm 0.0005$ \\
\hline$R_{p}\left(R_{E}\right)$ & $11.3 \pm 1.0$ & $R_{p}\left(R_{E}\right)$ & $2.87 \pm 0.30$ \\
\hline$b$ & $0.36 \pm 0.07$ & $b$ & $0.28 \pm 0.25$ \\
\hline$i(\mathrm{deg})$ & $89.6 \pm 1.3$ & $i(\operatorname{deg})$ & $89.71 \pm 0.29$ \\
\hline$M^{1 / 3} / R_{S}$ & $0.90 \pm 0.13$ & $M^{1 / 3} / R_{s}$ & $0.88 \pm 0.15$ \\
\hline$l d_{1}$ & $0.348 \pm 0.056$ & $l d_{1}$ & $0.371 \pm 0.087$ \\
\hline$l d_{2}$ & $1.03 \pm 0.19$ & $l d_{2}$ & $1.04 \pm 0.23$ \\
\hline KIC 11442793 g (KOI 351.02) & \multicolumn{3}{|c|}{ KIC 11442793 c } \\
\hline Period (days) & $210.60697 \pm 0.00043$ & Period (days) & $8.719375 \pm 0.000027$ \\
\hline Epoch (HJD-2454833) & $147.0364 \pm 0.0014$ & Epoch (HJD-2454833) & $139.5687 \pm 0.0023$ \\
\hline Duration (hr) & $12.593 \pm 0.045$ & Duration (hr) & $4.41 \pm 0.18$ \\
\hline$a / R_{s}$ & $127.3 \pm 4.1$ & $a / R_{S}$ & $16.0 \pm 0.8$ \\
\hline$a(\mathrm{AU})$ & $0.71 \pm 0.08$ & $a(\mathrm{AU})$ & $0.089 \pm 0.012$ \\
\hline$R_{p} / R_{s}$ & $0.0615 \pm 0.0011$ & $R_{p} / R_{s}$ & $0.0091 \pm 0.0003$ \\
\hline$R_{p}\left(R_{E}\right)$ & $8.1 \pm 0.8$ & $R_{p}\left(R_{E}\right)$ & $1.19 \pm 0.14$ \\
\hline$b$ & $0.45 \pm 0.10$ & $b$ & $0.09 \pm 0.20$ \\
\hline$i(\operatorname{deg})$ & $89.80 \pm 0.06$ & $i(\mathrm{deg})$ & $89.68 \pm 0.74$ \\
\hline$M^{1 / 3} / R_{S}$ & $0.84 \pm 0.14$ & $M^{1 / 3} / R_{S}$ & $0.90 \pm 0.16$ \\
\hline$l d_{1}$ & $0.34 \pm 0.10$ & $l d_{1}$ & $0.40 \pm 0.20$ \\
\hline$l d_{2}$ & $0.98 \pm 0.10$ & $l d_{2}$ & $1.21 \pm 0.26$ \\
\hline KIC $11442793 \mathrm{f}$ & \multicolumn{3}{|c|}{ KIC $11442793 b$} \\
\hline Period (days) & $124.9144 \pm 0.0019$ & Period (days) & $7.008151 \pm 0.000019$ \\
\hline Epoch (HJD-2454833) & $254.704 \pm 0.014$ & Epoch (HJD-2454833) & $137.6906 \pm 0.0017$ \\
\hline duration $(\mathrm{hr})$ & $10.94 \pm 0.25$ & Duration (hr) & $3.99 \pm 0.15$ \\
\hline$a / R_{s}$ & $86.4 \pm 9.7$ & $a / R_{S}$ & $13.2 \pm 1.8$ \\
\hline$a(\mathrm{AU})$ & $0.48 \pm 0.09$ & $a(\mathrm{AU})$ & $0.074 \pm 0.016$ \\
\hline$R_{p} / R_{s}$ & $0.0220 \pm 0.0022$ & $R_{p} / R_{s}$ & $0.0100 \pm 0.0005$ \\
\hline$R_{p}\left(R_{E}\right)$ & $2.88 \pm 0.52$ & $R_{p}\left(R_{E}\right)$ & $1.31 \pm 0.17$ \\
\hline$b$ & $0.35 \pm 0.40$ & $b$ & $0.13 \pm 0.32$ \\
\hline$i(\mathrm{deg})$ & $89.77 \pm 0.31$ & $i(\operatorname{deg})$ & $89.4 \pm 1.5$ \\
\hline$M^{1 / 3} / R_{s}$ & $0.84 \pm 0.20$ & $M^{1 / 3} / R_{s}$ & $0.85 \pm 0.21$ \\
\hline$l d_{1}$ & $0.360 \pm 0.068$ & $l d_{1}$ & $0.378 \pm 0.060$ \\
\hline$l d_{2}$ & $1.01 \pm 0.18$ & $l d_{2}$ & $1.11 \pm 0.20$ \\
\hline \multicolumn{4}{|l|}{ KIC 11442793 e } \\
\hline Period (days) & $91.93913 \pm 0.00073$ & & \\
\hline Epoch (HJD-2454833) & $134.3127 \pm 0.0063$ & & \\
\hline Duration (hr) & $9.71 \pm 0.19$ & & \\
\hline$a / R_{s}$ & $74.7 \pm 4.3$ & & \\
\hline$a(\mathrm{AU})$ & $0.42 \pm 0.06$ & & \\
\hline$R_{p} / R_{s}$ & $0.0203 \pm 0.0005$ & & \\
\hline$R_{p}\left(R_{E}\right)$ & $2.66 \pm 0.29$ & & \\
\hline$b$ & $0.27 \pm 0.22$ & & \\
\hline$i(\mathrm{deg})$ & $89.79 \pm 0.19$ & & \\
\hline$M^{1 / 3} / R_{S}$ & $0.87 \pm 0.15$ & & \\
\hline$l d_{1}$ & $0.360 \pm 0.049$ & & \\
\hline$l d_{2}$ & $1.05 \pm 0.17$ & & \\
\hline
\end{tabular}

Note. Values calculated for $R_{s}=1.2 \pm 0.1 R_{\odot} ; R_{\odot}=696342 \mathrm{~km}$ and $R_{E}=6378 \mathrm{~km}$.

curve with the obtained values of the individual epochs. This method does not converge in the case of planets $b$ and $c$ because of the low $\mathrm{S} / \mathrm{N}$ of their transit signal. Therefore, for planets $\mathrm{b}$ and c, we fix the period; we do not fit for the epochs, but we do apply the stellar activity correction for each individual transit described above.

A detailed description of the modeling of the planetary parameters applied here can be found in Csizmadia et al. (2011).
We used the publicly available short cadence Kepler light curves. For candidates b, c, d, e, and f, we binned the light curves (we formed 2000 binned points in the $\pm 2 D$ vicinity of the midtransit, $D$ being the transit duration), while for candidates $g$ and $\mathrm{h}$, we used the original short cadence photometric points. We used the Mandel \& Agol (2002) transit model. This model gives the light loss of the star due to the transit of an object as a function of their size ratio $(\mathrm{k})$, mutual sky-projected distance (denoted 
by $\delta$ ), and the limb darkening coefficients of the transited star $\left(l d_{1}=u_{1}+u_{2}\right.$, and $\left.l d_{2}=u_{1}-u_{2}\right)$.

Following Csizmadia et al. (2013), we determined the limb darkening coefficients from the light curve instead of using theoretical predictions. This fit was first applied to planet h, which has the largest transit depth, i.e., the highest S/N. Having obtained the values of the limb darkening coefficients, we set the limb darkening coefficients at the value obtained from the fit of planet g's transit light curve, but we allowed them to vary within the uncertainties of the determined values.

Since we do not have any radial velocity measurements, nor occultations, nor phase-curves of any of these seven planets, we had no a priori information about eccentricities and arguments of periastron. Therefore, we could not calculate the sky-projected distance of the stellar and planetary centers in the usual way (e.g., Giménez 2006). Instead, we fitted the duration of the transit, the epoch $\left(t_{0}\right)$, the period $(P)$, the impact parameter (b), and the planet-to-stellar radii ratio $\left(k=R_{p} / R_{s}\right)$. The skyprojected mutual distance of the star and the planet were then calculated with the formula

$$
\delta \approx \sqrt{b^{2}+\left[(1+k)^{2}-b^{2}\right]\left(\frac{t-t_{0}}{P}\right)^{2}},
$$

where $t$ is the time. We checked the validity of this latter formula via numerical experiments, and we found that in our cases, it yields a very good agreement with the theoretical value in the vicinities of transits. No mutual transit event was modeled. For the optimization, a genetic algorithm process described in Csizmadia et al. (2011) was used, and the results were refined by a Simulated Annealing algorithm which was also used for the error estimation. The reported uncertainties in Table 3 are $1 \sigma$ uncertainties.

We report the modeled values of $k$ and $b$ in Table 3 with their respective uncertainties for each of the seven candidates in the system. Once $k, b, D, P$ became known from the modeling procedure, the value of the scaled semi-major $\left(a / R_{s}\right)$ for circular orbits can then be calculated as

$$
\frac{a}{R_{s}}=\frac{1}{\pi} \frac{P}{D} \sqrt{(1+k)^{2}-b^{2}} .
$$

We then calculated the scaled semi-major axes for every planet in the system assuming circular orbits (see Table 3). Rewriting Kepler's third law, we obtained the stellar density parameter (neglecting the mass of the planet):

$$
\frac{M^{1 / 3}}{R_{S}}=\left(\frac{3 \pi}{G P^{2}}\right)^{1 / 3} \frac{a}{R_{S}}
$$

or equivalently

$$
\begin{aligned}
\frac{M^{1 / 3}}{R_{s}}= & \left(\frac{3}{G P D}\right)^{1 / 3}\left[(1+k)^{2}-b^{2}\right]^{(3 / 2)} \\
& \times\left(\frac{1-e^{2}}{1+e^{2}-2 e \sin \omega}\right)^{3 / 2} .
\end{aligned}
$$

We also report the density parameter derived from every candidate in Table 3. Figure 3 shows the modeling of the photometric light curves and the model residuals for each planet in graphical form.

\subsection{Analysis of the Geometry of the Transits}

One argument supporting the hypothesis that all of these planet candidates orbit the same star comes from the modeling of the planetary parameters. The inclinations and stellar densities $\left(M^{1 / 3} / R_{S}\right)$ shown in Table 3 were calculated independently for each planet. They are all compatible to each other, and the density is compatible with the value obtained independently for the stellar parameters in Section 2.

We can also provide another geometrical argument supporting the former hypothesis using the measured durations and periods of the transiting planets. These are obtained from a pure geometrical fit to the transits, independently of the planetary modeling techniques. This argument has previously been used in the literature to support the hypothesis that multiple candidate systems actually orbit the same star (Chaplin et al. 2013). Figure 4 shows how the transit durations distribute as a function of planetary periods. If all planets orbit the same star in circular, coplanar orbits, the transit duration $D$ should relate to the orbital period $P$ through Kepler's third law:

$$
D=\frac{\alpha}{\pi} P^{1 / 3} \sqrt{1-\left(\frac{\cos i^{2}}{\alpha} P^{4 / 3}\right)},
$$

where $\alpha=\left(3 \pi / G / \rho_{s}\right)^{1 / 3}$, and $\rho_{s}$ is the density of the star. If $D$ and $P$ are in days, the best fit to the data gives a value of $\alpha=0.23$ and $i=90^{\circ}$, compatible with the values obtained from the stellar and planetary modeling. Note that the fit is not a physical solution, because all planetary orbits do not need to be exactly coplanar. However, they are compatible with all planets orbiting the same star in nearly edge-on aligned orbits, which supports our hypothesis that all planets orbiting the same star.

\section{TRANSIT TIMING VARIATIONS}

The analysis of the TTVs has proven to be a versatile tool to confirm the planetary nature of transiting candidates (Ford et al. 2011). Typically, TTVs have amplitudes of several minutes (with some exceptional cases like KOI 142, Nesvorný et al. 2013, with an amplitude of $12 \mathrm{hr}$ ) and typically periods one order of magnitude larger than the orbital period of the planet involved (Mazeh et al. 2013).

Figure 5 shows the individual transits and Figure 6 the $O-C$ diagram for candidate $\mathrm{g}$. The transit corresponding to epoch 7 (epoch 1 being the value provided in Table 3 ) has a displacement of $25.7 \mathrm{hr}$ with respect to its expected position. This abrupt change is due to a change in the osculating orbital elements produced by the gravitational interaction with other objects in the system, possibly candidate h (see Section 6). Most surveys of TTVs expect discovering periodic modulations of the timing perturbations (see a derivation of the searched expression in Lithwick et al. 2012 and the series of papers Ford et al. 2011, 2012a, 2012b; Steffen et al. 2012a, 2012b, 2013; Fabrycky et al. 2012; Mazeh et al. 2013). However, nonperiodic sudden changes of the orbital elements, corresponding to irregular behavior such as the one displayed by planet $\mathrm{g}$, have been theoretically described (for example, though in a different context, Holman \& Murray 2005), but we believe that we report an observational example for the first time.

In addition to the change in the osculating elements, it is interesting to discuss the other transit events recorded for candidate $\mathrm{g}$ separately. The depth and the duration of transit events 1,2 , and 3 changes significantly. One can speculate that the perturbations seen around these transits are morphologically 

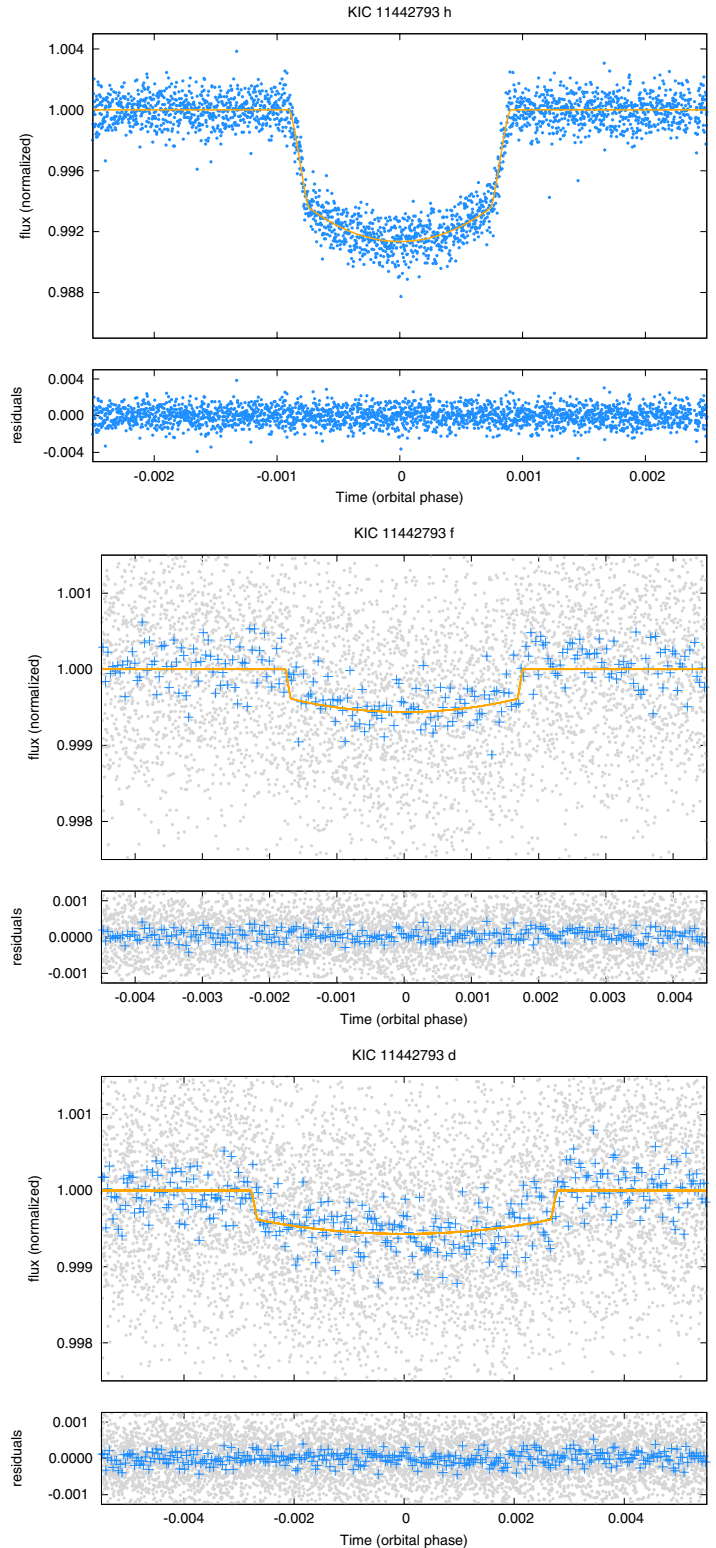

KIC $11442793 \mathrm{~b}$

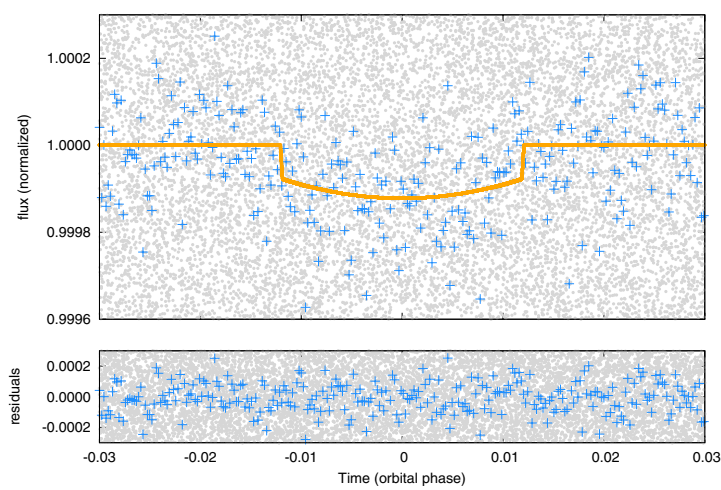

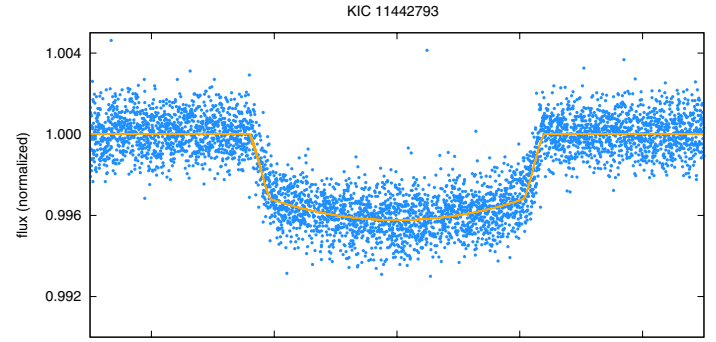
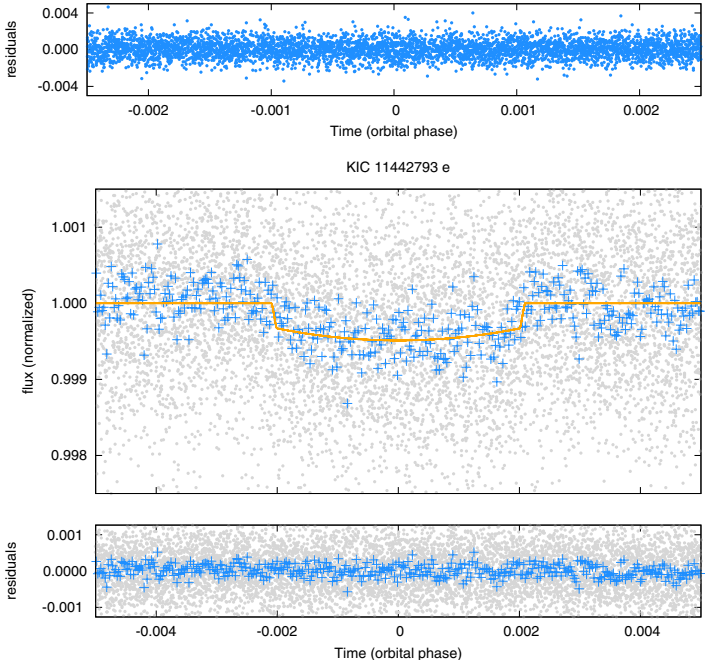

KIC 11442793
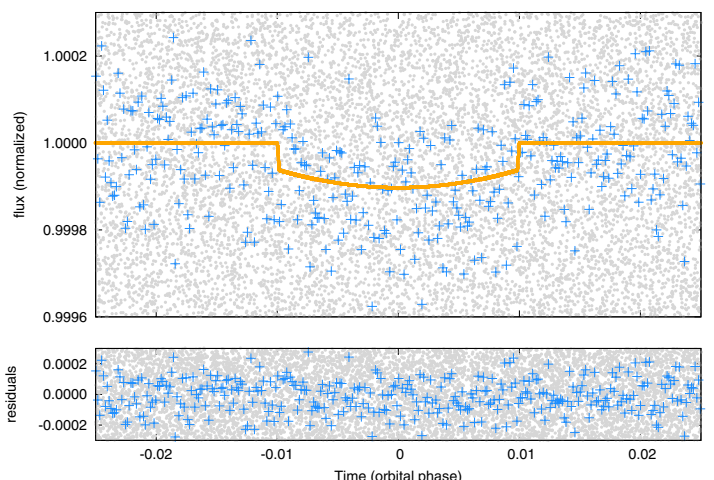

Figure 3. Filtered light curve of KIC 11442793 folded at the period of the different planets. For planets b and c, the light curve has been binned to help the eye. The orange solid line shows the light curve fit (Table 3). The lower panels show the residuals of the light curve fit, respectively.

(A color version of this figure is available in the online journal.)

equivalent to those produced by a moon around the planet (Sartoretti \& Schneider 1999; Kipping et al. 2013b). This hypothesis is further discussed in Section 7.1. We do not have enough evidence to prove that these perturbations are produced by a moon and until we have constraints on the planetary masses, we cannot assess the stability of moons around candidate g. We note, just for completion, that a moon could not be responsible in any case for the abrupt change in the osculating orbital elements 


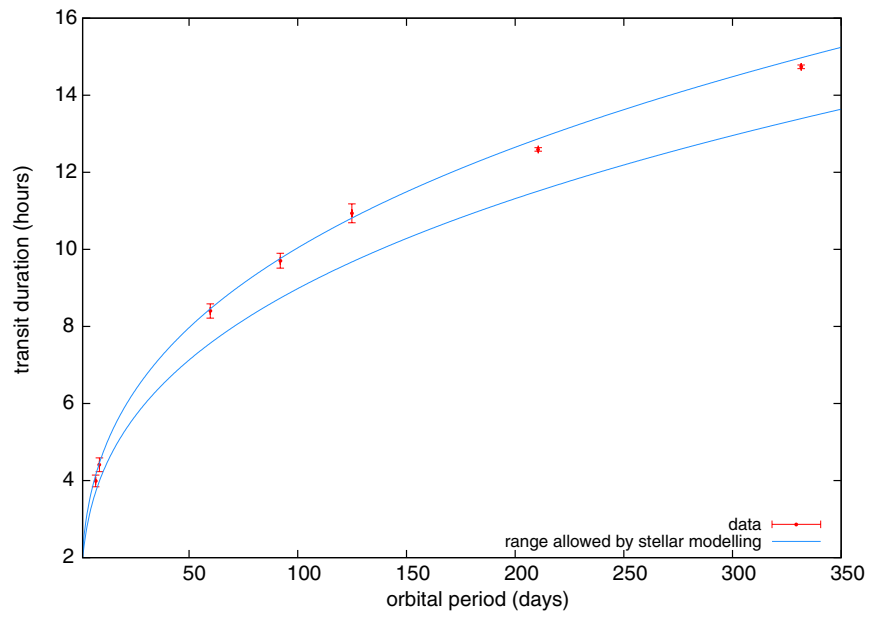

Figure 4. Transit duration of each planet as a function of their orbital period. The observed values are compatible with the seven planets orbiting a star whose density is that given by the stellar and planetary parameter modeling on edge-on aligned orbits. The range allowed by the modeling of the stellar parameters is indicated with the continuous blue lines.

(A color version of this figure is available in the online journal.)

displayed in transit event 7 . The amplitudes of the perturbations produced by moons are typically only a few seconds (Cabrera 2010; Kipping 2009a, 2009b).

The available data set for KIC 11442793 does not allow us to do an unambiguous determination of the planetary masses from the analysis of the TTVs. Candidates $\mathrm{b}$ and $\mathrm{c}$ are too small and too close to the detection limit to measure any reliable TTV amplitude (see Figure 6), which is not unusual in the case of low-mass planets in compact systems (see the case of CoRoT$7 b$ Léger et al. 2009). The TTVs of candidates $d$ and e are compatible with zero within the limits of our current modeling (see Figure 6). There are only five full transits observed from the nine expected transits of candidate $f$ due to some unfortunate coincidence of observing interruptions with the expected transit positions. However, there is a significant signal in the available $O-C$ diagram, which means that candidate $\mathrm{f}$ is interacting dynamically with other objects in the system.

Candidate $\mathrm{g}$ shows six transits in the available data set (expected seven) and candidate $\mathrm{h}$ shows three transits (expected five), less than expected due to the interruptions of the photometric record (duty cycle is $82 \%$ ). However, candidates $g$ and $\mathrm{h}$ show both significant TTVs and transit duration variations; consequently, we deduce that they are interacting dynamically.

\section{DYNAMICAL STUDY}

\subsection{Analysis with a Numerical Integrator}

We have done a stability analysis of the system with the orbital dynamics integrator Mercury (Chambers 1999). The system is only stable if candidates $\mathrm{g}$ and $\mathrm{h}$ have masses below some Jupiter units (typically, less than 5 Jupiter masses). Therefore, we conclude that $g$ and $h$ are planets because they interact gravitationally and their long-term dynamical stability is only guaranteed if these bodies have planetary masses.

The Mercury numerical analysis of the planetary system reveals that objects $\mathrm{d}, \mathrm{e}$, and $\mathrm{f}$ are in stable orbits only if those are very circular (typically, less than $3 \%$ for mass values of 10 Earth masses, representative of 2.5 Earth radii super-Earths) and planetary masses (less than the mass of Jupiter). Therefore, we conclude that these three must also be planets. Actually, the requirement of the circularity of their orbits implies that, for the system to be stable, the mean motion resonance (MMR) has to play a role to guarantee the survival of the system.

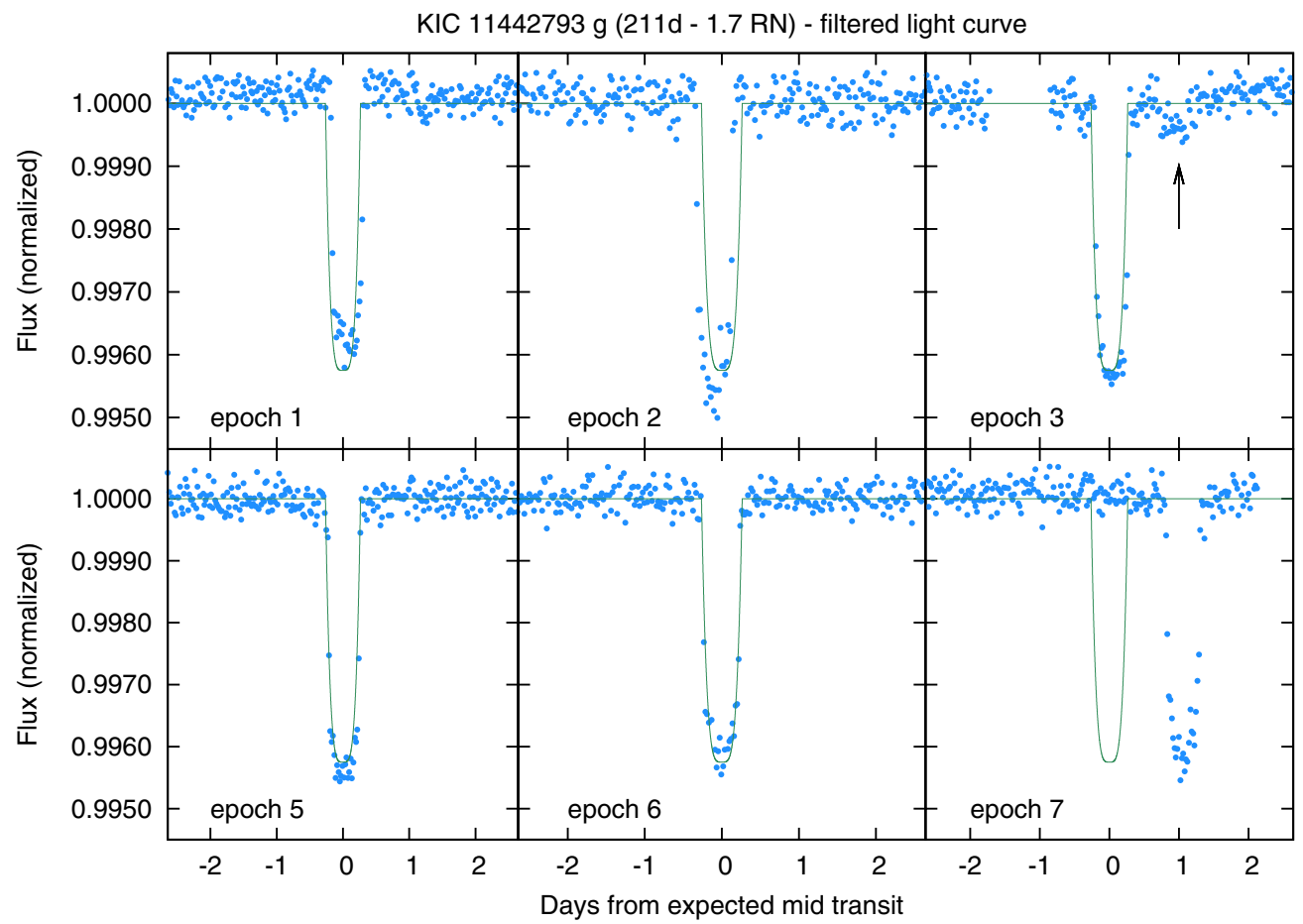

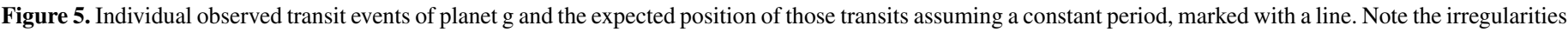

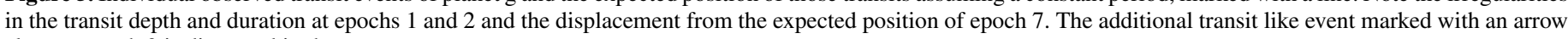
close to epoch 3 is discussed in the text.

(A color version of this figure is available in the online journal.) 

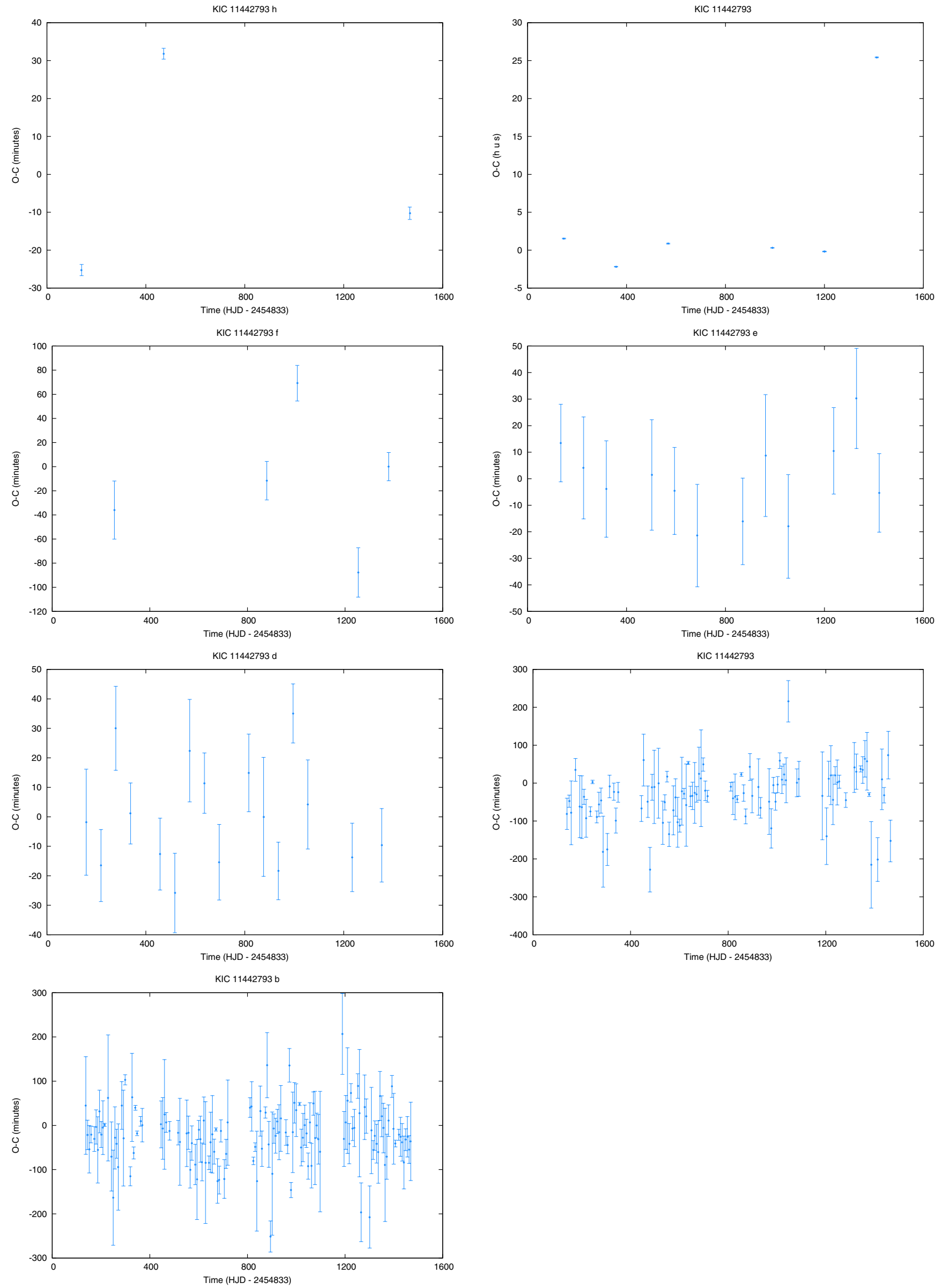

Figure 6. Transit timing variations of the different planets. Observed midtimes of planetary transits $(\mathrm{O})$ minus calculated linear ephemeris $(\mathrm{C})$ are plotted with $1 \sigma$ uncertainties.

(A color version of this figure is available in the online journal.) 
We did not see any sign that candidates $b$ and $c$ interact dynamically with the other planets in the system because the low S/N of the transit light curves. The Mercury numerical analysis reveals that their orbits are, in principle, only stable if the objects have planetary masses.

\subsection{A First Dynamical Study}

We estimated the masses of the seven planets considering their sizes and assuming representative mean densities for each planetary class (gas giant, ice giants, large and small superEarth) as follows: planet $m_{h}=0.8 M_{\text {Jupiter }}, m_{g}=0.7 M_{\text {Neptune }}$, planets $m_{f} \sim m_{e} \sim m_{d} \sim 10 M_{\text {Earth }}$ and planets $m_{c} \sim m_{b} \sim$ $3 M_{\text {Earth }}$. Given the periods and the estimated semi-major axes, we can compute their separation in terms of Hill radii. Using the formula given below (Chambers et al. 1996):

$$
H=\left(\frac{m_{1}+m_{2}}{3}\right)^{\frac{1}{3}} \frac{a_{1}+a_{2}}{2},
$$

we get the following numbers for the separation of neighboring planets in Hill radii:

$$
\begin{aligned}
& g-h: 5, h-f: 11, f-e: 5, e-d: 10, \\
& d-c: 47, \text { and } c-b: 10 .
\end{aligned}
$$

This indicates the stability of the different subsystem given they are moving in almost circular orbits. The inner planets b, c, $\mathrm{d}$, e, and $\mathrm{f}$ in particular are relatively safe in their orbits, which is evident from their Hill radii. It is interesting to note that the innermost two planets are in a 4:5 MMR; the two massive outer ones are not far from a 5:8 MMR. It is also worth mentioning that the three planets $\mathrm{d}$, e, and $\mathrm{f}$ are close to the interesting Laplace resonance, which is known to happen for the motion of the three Galilean moons of Jupiter (Io, Europa, and Ganymede) but also for the three moons of Uranus (Miranda, Ariel, and Umbriel, e.g., Ferraz-Mello 1979):

$$
\frac{1}{P_{d}}-\frac{3}{P_{e}}+\frac{2}{P_{f}} \sim 0 .
$$

Because the inner system consisting of super-Earth planets is quite stable, we concentrate on the dynamics of the planets $h$ and $g$. The stability of this extrasolar planetary system seems to depend on the stability of the orbits of these two outer gas giants, which may have even eccentric orbits given the relative distance to the star. So we tried to find borders for stable motion of the two outer gas giants using the results of long-term integrations up to $10^{7}$ yr. $^{8}$

It turned out that inside the domain of motion for $e_{h}<0.095$ and $e_{g}<0.025$, the orbits of the two outer planets are regular with only slight periodic changes in the eccentricities (see Figure 8, lower right graph). The closeness to the 8:5 MMR is not destroying their stability; an additional resonance appears for the motions of the perihelia of $g$ and $h$. This secular resonance is depicted in Figure 7, where the 1:1 resonance of the motion of $\omega_{g}$ and $\omega_{h}$ with a period of about $1.7 \times 10^{4} \mathrm{yr}$ is visible.

Close to the edge of the stable region, we have an intermediate region where stable and unstable orbits are very close to each other (see Figure 8). In this domain, we find the so-called sticky orbits-a well-known phenomenon of dynamical systems (e.g.,

\footnotetext{
8 For the integration method, we used the a high-precision LIE-integrator
} with automatic step (e.g., Hanslmeier \& Dvorak 1984).
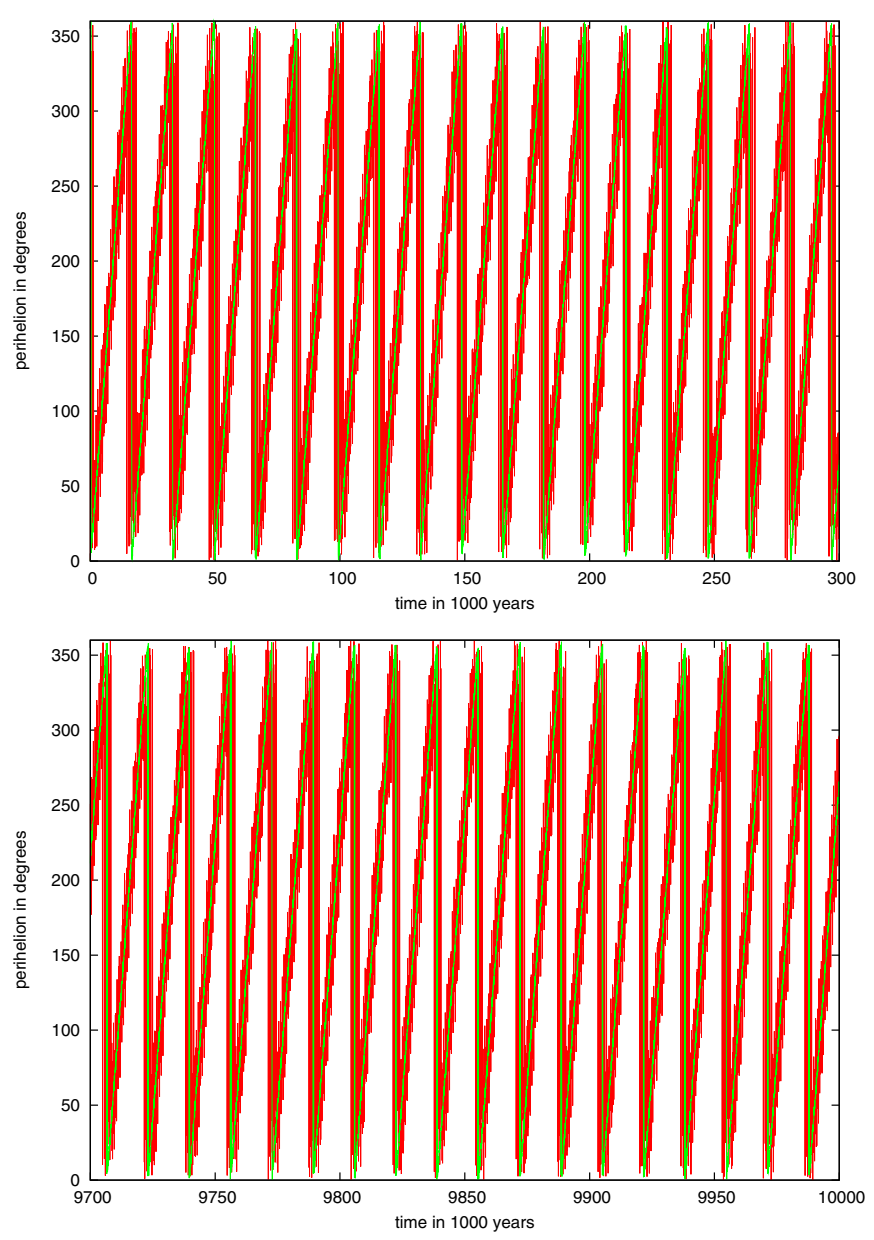

Figure 7. Perihelion motion of the two outer planets $h$ and $g$ for initial conditions in the stable domain (see the text). Out of the whole integration time of $10^{7} \mathrm{yr}$, we show the first $3 \times 10^{5}$ (upper graph) and the last $3 \times 10^{5} \mathrm{yr}$ (lower graph). The strong coupling in a 1:1 secular resonance of the perihelion motion with a period of around $17 \times 10^{4}$ is clearly visible.

(A color version of this figure is available in the online journal.)

Dvorak et al. 1998), an orbit there is "stuck" to an invariant torus in phase space and then escapes through a hole of the last KAMtorus. ${ }^{9}$ In the respective figure, e show three such examples where a small shift in eccentricity of planet $\mathrm{h}(\Delta e=0.005)$ causes such a different dynamical behavior of an orbit.

We need to explain the large TTV for planet g. The answer is visible in Figure 9, where one can see the relatively large variations of the semi-major axis of this planet even for a time scale of years. This change can lead to a change in the period which achieves values up to a day from one transit to another one, comparable to the changes observed in the Kepler data.

But the system is even more complex. Because planet $g$ is in 5:3 MMR with planet $f$ and this one is in the formerly mentioned Laplace resonance (with the planets e and d), the stability limit for the eccentricities of all planets is very small. Integrating the "complete" system ${ }^{10}$ it is only stable well before the stability limit mentioned above for the eccentricities of planets $h$ and $g$; the absolute limit for a stable system is $e<0.001$ for all five outer planets!

We conclude from the preliminary dynamical study of this seven planet system that, with the actual parameters determined,

\footnotetext{
9 KAM stands for Kolmogorov-Arnold-Moser.

10 One can ignore the two innermost super-earth-planets-so we integrated the star plus the five outer planets.
} 

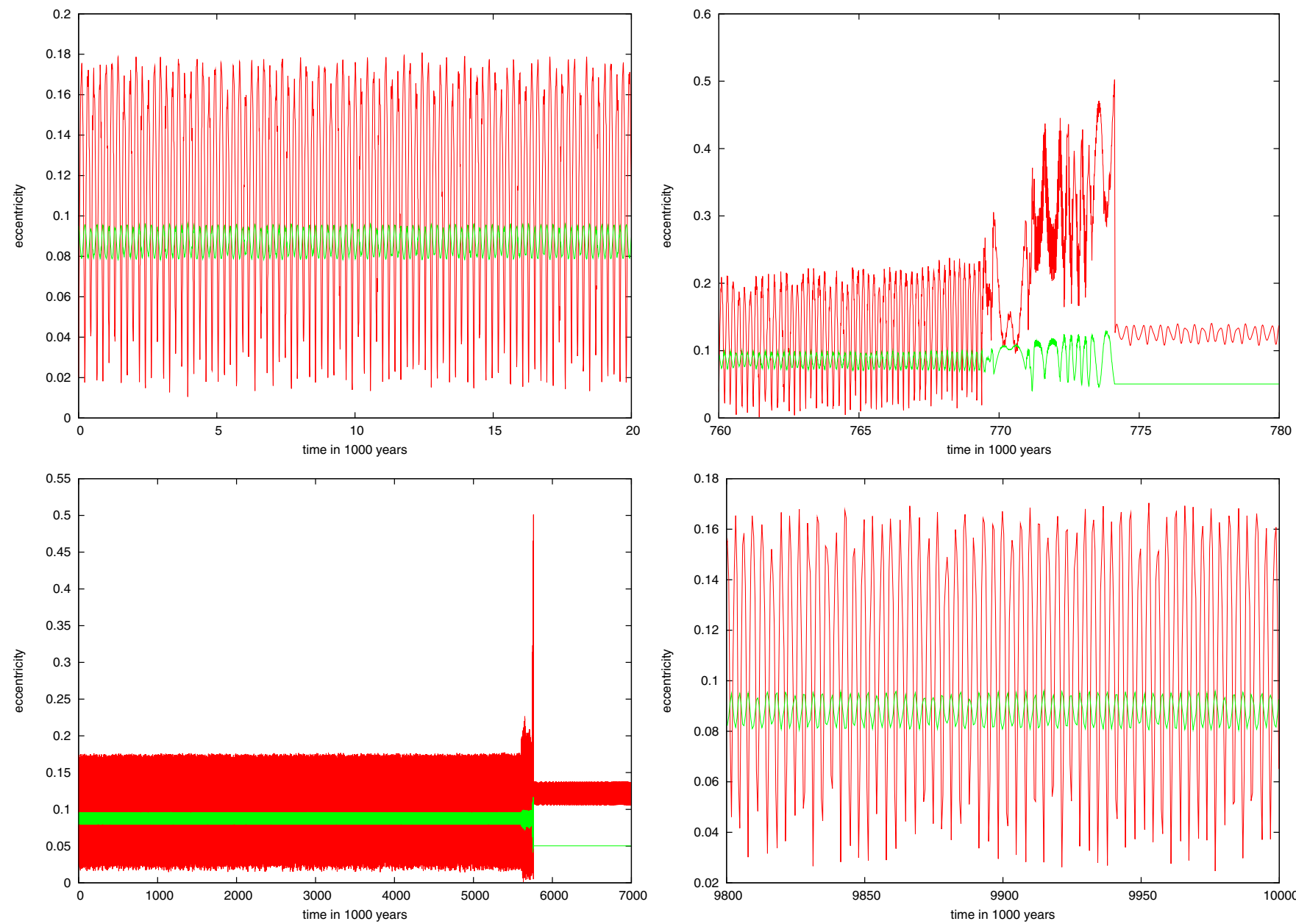

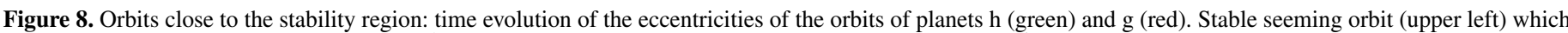

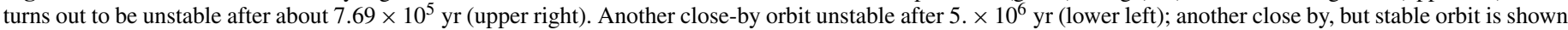
(lower right). For details, see text.

(A color version of this figure is available in the online journal.)
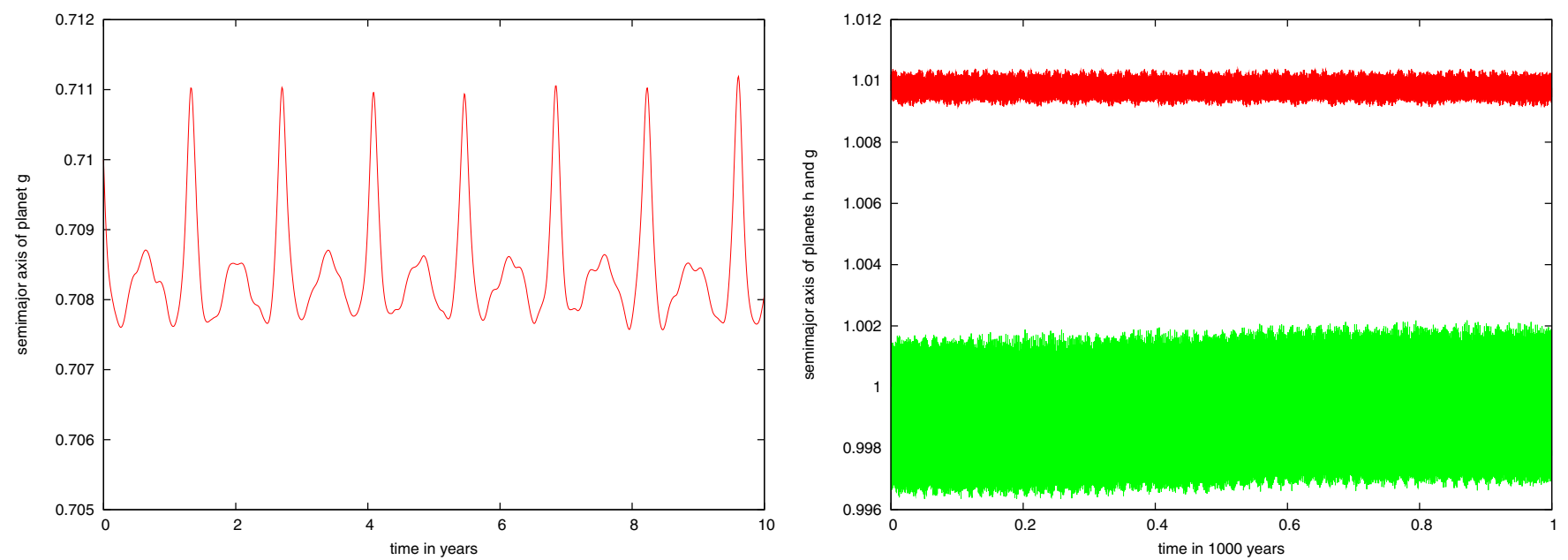

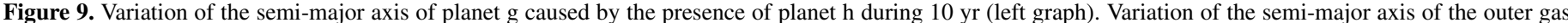

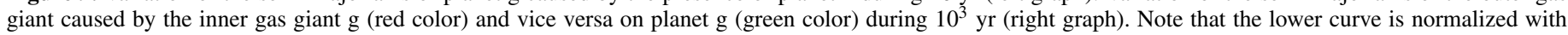
respect to the semi-major axis of $a=0.71 \mathrm{AU}$ of planet $\mathrm{g}$.

(A color version of this figure is available in the online journal.)

it is quite close to instability. Consequently, the parameters like the masses and the semi-major axes need some revision after a deeper dynamical study, out of the scope of this paper. Even in our solar system, where the orbital parameters are well determined, the issue of the long-term stability is debated (e.g., Laskar 1994, 2008), and the influence of many different resonances is complex. We are currently working on that dynamical study (R. Dvorak et al. in preparation). 


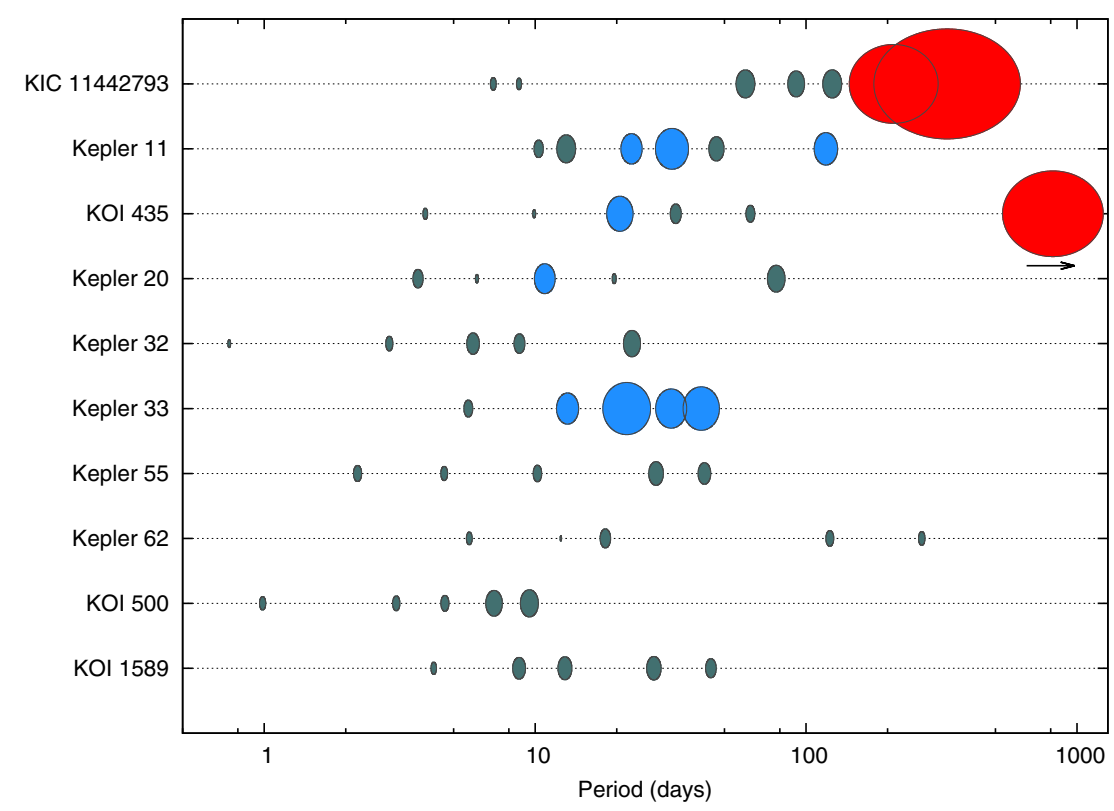

Figure 10. Comparison of different multiple systems: Kepler-11 (Lissauer et al. 2011), KOI-435 (Ofir \& Dreizler 2013), Kepler-20 (Gautier et al. 2012 ; Fressin et al. 2012), Kepler-32 (Fabrycky et al. 2012), Kepler-33 (Lissauer et al. 2012), Kepler-55 (Steffen et al. 2013), Kepler-62 (Borucki et al. 2013), KOI-500 (Xie 2013; Wu \& Lithwick 2013), and KOI-1589 (Xie 2013; Wu \& Lithwick 2013). Color codes separate Earth and super-Earth planets (up to 4 Earth radii, shown in green), Neptune-sized planets (between 4 and 8 Earth radii, shown in blue), and gas giants (larger than 8 Earth radii, shown in red).

(A color version of this figure is available in the online journal.)

\section{KIC 11442793 IN THE CONTEXT OF OTHER MULTIPLANET SYSTEMS}

Models of planet formation include theories about planet-planet scattering followed by tidal circularization (Rasio \& Ford 1996; Lin \& Ida 1997; Chatterjee et al. 2008; Beaugé \& Nesvorný 2012). Another possible mechanism of planet formation builds planets at relatively large distances from the star, and later these planets migrate inward through a disk (Goldreich \& Tremaine 1980; Lin et al. 1996; Ward 1997; Murray et al. 1998). The first mechanism does not likely form compact multiple systems such as the ones observed by Kepler (Batalha et al. 2013), characterized by being compact and having low relative inclination orbits (Fang \& Margot 2012; Tremaine \& Dong 2012). Different mechanisms have been proposed to explain the origin of the latter systems. One promising possibility is in-situ formation (see, for example, Chiang \& Laughlin 2013; Chatterjee \& Tan 2013), including the observed feature that many of those systems have planets orbiting close to, but not exactly at, MMRs (Lithwick \& Wu 2012; Petrovich et al. 2013).

In Figure 10, we show a schematic view of the periods and relative sizes of nine multiple transiting planetary systems discovered by Kepler with five transiting planets or more, together with the planetary system reported in this paper. There are also multiple systems discovered by radial velocity hosting six or more planets, like GJ 667C (Anglada-Escudé et al. 2013), HD 40307 (Tuomi et al. 2013), or HD 10180 (Lovis et al. 2011). However, their orbital properties and even their existence is not as secure as those of transiting candidates. For example, consider the case of the system GJ 581 (Hatzes 2013) or the discussion in the literature if HD 10180 is orbited by six (Feroz et al. 2011), seven (Lovis et al. 2011), or even nine (Tuomi 2012) planets. Therefore, we limit ourselves in Figure 10 to the discussion of multiple transiting systems. Among the systems shown, KIC 11442973 presented here is the only one showing a clear hierarchy like our solar system. Additionally, only
KIC 11442973 and KOI 435 include a giant planet larger than 10 Earth radii. Such systems are typically more difficult to form because giant planets tend to excite the eccentricity of less massive planets during the migration processes, compromising the long-term stability of the system (see, for example, Raymond et al. 2008).

Note that there are two additional known systems simultaneously hosting super-Earths and gas giants, but these two systems orbit M dwarfs, and only the second example is a compact system. GJ 676A (Anglada-Escudé \& Tuomi 2012) hosts up to four planets, including one super-Earth in a 3.6 day orbit and one planet of 5 Jupiter masses in a 1050 day orbit. GJ 876 (Rivera et al. 2010) is also an M-dwarf hosting one super-Earth of 6 Earth masses at 1.9 day orbital period, a 0.7 Jupiter masses planet at 20 days, a 2.3 Jupiter masses planet at 61 days, and a 14 Earth masses planet in a 124 day orbit. However, KIC 11442793 is a late $\mathrm{F} /$ early $\mathrm{G}$ solar-like star, hosting a more complex system where dynamical interactions play an important role in the long-term stability of the system.

\subsection{About the Possible Existence of Moons in the Planetary System}

In the previous section, we have discussed the possibility that KIC $11442793 \mathrm{~g}$ hosts a moon. Figure 5 shows that the transit epochs 1,2, and 3 show features morphologically equivalent to an exomoon orbiting the planet (Sartoretti \& Schneider 1999; Szabó et al. 2006; Kipping 2011). However, considering the distance between the transit epoch 3 and the moon-like event marked with an arrow in Figure 5, the estimated projected distance between the planet and the exomoon candidate would be orbiting close to the Hill radius of the planet, which is too far away to guarantee the long-term stability of the satellite, usually limited to a distance of one-third (Barnes \& O'Brien 2002) to one-half (Domingos et al. 2006) of the planetary Hill sphere. With the current data set, we cannot exclude that the 
event marked with an arrow in Figure 5 is caused by instrumental residuals. However, the distorted shape features of transits 1 and 2 cannot be explained simply by the impact of stellar activity, and their origin remains unclear. Space surveys have regularly been used to rule out the presence of moons around extrasolar planets (Pont et al. 2007; Deeg et al. 2010). So far, the most extensive search for exomoons (Kipping et al. 2012) has taken advantage of the simultaneous change in the transit timing and transit duration changes produced by the hypothetical satellites (Kipping 2009a, 2009b). However, until now, only negative results have been reported (Kipping et al. 2013b, 2013a). A possible reason for this lack of success is that searches have been limited to isolated, typically non-giant planets. Even if these systems are formed by planet-planet scattering, they are unlikely to maintain the moons during their formation process (Gong et al. 2013). In turn, migration tends to remove moons from planetary systems (Namouni 2010). Therefore, in-situ formed compact systems could be more prone to host exomoons in long timescales.

\section{SUMMARY}

We report the discovery of a planetary system with seven transiting planets with orbital periods in the range from 7 to 330 days ( 0.074 to $1.01 \mathrm{AU})$. The system is hierarchical, the two innermost planets have sizes close to Earth and their period ratio is within $0.5 \%$ of the $4: 5 \mathrm{MMR}$. The three following planets are super-Earths with sizes between 2 and 3 Earth radii whose periods are close to a 2:3:4 chain. From the observational data set, we cannot determine their masses or the value of their mean longitudes but the ratio of their mean motions is close to a Laplace resonance. The outermost planets are two gas giants at distances of 0.7 and 1.0 AU. There are other systems of superEarths, discovered either by radial velocity or by transit, which show some similarities, for example GJ 876 (Rivera et al. 2010) or KOI 152 (Wang et al. 2012), but these systems only contain super-Earths, while KIC 11442793 is a hierarchical system. As a singularity among the other multiple systems found by Kepler or radial velocity, KIC 11442793 contains a gas giant planet similar to Jupiter orbiting at $1 \mathrm{AU}$. Systems with super-Earths close to a Laplace resonance are also believed to be frequent (Chiang \& Laughlin 2013) but this particular system poses new challenges due to the presence of the gas giants $g$ and $h$, which seem to have the most intense gravitational interaction measured among extrasolar planets so far (25.7 hr of change in the ephemeris). If Kepler cannot continue the follow up of this system (Cowen 2013), the follow-up of the Earth and super-Earth planets of this system will be challenging in the near future, as they are beyond reach for CHEOPS (Broeg et al. 2013) or TESS (Ricker et al. 2010). Only PLATO (Rauer \& Catala 2011) will be able to study their evolution in detail. However, the gas giants $\mathrm{g}$ and f produce $0.5 \%$ and $0.8 \%$ transits, which should be observable from the ground, which makes this system an attractive target for future follow-up studies.

We are grateful to É. Bálint, Ph. von Paris, and M. Godolt for useful discussions concerning this paper. This paper includes data collected by the Kepler mission. Funding for the Kepler mission is provided by the NASA Science Mission directorate. Some/all of the data presented in this paper were obtained from the Mikulski Archive for Space Telescopes (MAST). STScI is operated by the Association of Universities for Research in Astronomy, Inc., under NASA contract NAS5-26555. Support for
MAST for non-HST data is provided by the NASA Office of Space Science via grant NNX09AF08G and by other grants and contracts. The research leading to these events has received funding from the European Union Seventh Framework Programme (FP7/2007-2013) under grant agreement No. 267251.

\section{REFERENCES}

Alapini, A., \& Aigrain, S. 2009, MNRAS, 397, 1591

Anglada-Escudé, G., \& Tuomi, M. 2012, A\&A, 548, A58

Anglada-Escudé, G., Tuomi, M., Gerlach, E., et al. 2013, A\&A, 556, A126

Baglin, A., Auvergne, M., Boisnard, L., et al. 2006, in COSPAR, Plenary Meeting, Vol. 36, 36th COSPAR Scientific Assembly, 3749

Barnes, J. W., \& O'Brien, D. P. 2002, ApJ, 575, 1087

Batalha, N. M., Rowe, J. F., Bryson, S. T., et al. 2013, ApJS, 204, 24

Batalha, N. M., Rowe, J. F., Gilliland, R. L., et al. 2010, ApJL, 713, L103

Beaugé, C., \& Nesvorný, D. 2012, ApJ, 751, 119

Borucki, W. J., Agol, E., Fressin, F., et al. 2013, Sci, 340, 587

Borucki, W. J., Koch, D., Basri, G., et al. 2010, Sci, 327, 977

Broeg, C., Fortier, A., Ehrenreich, D., et al. 2013, European Physical Journal Web of Conferences, 47, 3005

Cabrera, J. 2010, in EAS Publications Series, Vol. 42, ed. K. Gożdziewski, A. Niedzielski, \& J. Schneider (Cambridge: Cambridge Univ. Press), 109

Cabrera, J., Csizmadia, S., Erikson, A., Rauer, H., \& Kirste, S. 2012, A\&A, 548, A44

Chambers, J. E. 1999, MNRAS, 304, 793

Chambers, J. E., Wetherill, G. W., \& Boss, A. P. 1996, Icar, 119, 261

Chaplin, W. J., Sanchis-Ojeda, R., Campante, T. L., et al. 2013, ApJ, 766, 101

Chatterjee, S., Ford, E. B., Matsumura, S., \& Rasio, F. A. 2008, ApJ, 686, 580 Chatterjee, S., \& Tan, J. C. 2013, arXiv:1306.0576

Chiang, E., \& Laughlin, G. 2013, MNRAS, 431, 3444

Cowen, R. 2013, Natur, 497, 417

Csizmadia, S., Moutou, C., Deleuil, M., et al. 2011, A\&A, 531, A41

Csizmadia, S., Pasternacki, T., Dreyer, C., et al. 2013, A\&A, 549, A9

Cutri, R. M., Skrutskie, M. F., van Dyk, S., et al. 2003, 2MASS All Sky Catalog of Point Sources NASA/IPAC Infrared Science Archive, http://irsa.ipac.caltech.edu/applications/Gator

Deeg, H. J., Moutou, C., Erikson, A., et al. 2010, Natur, 464, 384

Domingos, R. C., Winter, O. C., \& Yokoyama, T. 2006, MNRAS, 373, 1227

Dvorak, R., Contopoulos, G., Efthymiopoulos, C., \& Voglis, N. 1998, P\&SS, 46, 1567

Fabrycky, D. C., Ford, E. B., Steffen, J. H., et al. 2012, ApJ, 750, 114

Fang, J., \& Margot, J.-L. 2012, ApJ, 761, 92

Feroz, F., Balan, S. T., \& Hobson, M. P. 2011, MNRAS, 415, 3462

Ferraz-Mello, S. 1979, Dynamics of the Galilean Satellites: An Introductory Treatise (Sao Paulo, Brazil, Instituto Astronomico e Geofisico)

Ford, E. B., \& Gaudi, B. S. 2006, ApJL, 652, L137

Ford, E. B., Fabrycky, D. C., Steffen, J. H., et al. 2012a, ApJ, 750, 113

Ford, E. B., Ragozzine, D., Rowe, J. F., et al. 2012b, ApJ, 756, 185

Ford, E. B., Rowe, J. F., Fabrycky, D. C., et al. 2011, ApJS, 197, 2

Fressin, F., Torres, G., Rowe, J. F., et al. 2012, Natur, 482, 195

Gandolfi, D., Alcalá, J. M., Leccia, S., et al. 2008, ApJ, 687, 1303

Gautier, T. N., III, Charbonneau, D., Rowe, J. F., et al. 2012, ApJ, 749, 15

Geem, Z. G., Kim, J. H., \& Loganathan, G. V. 2001, Simul, 76, 60

Giménez, A. 2006, A\&A, 450, 1231

Goldreich, P., \& Tremaine, S. 1980, ApJ, 241, 425

Gong, Y.-X., Zhou, J.-L., Xie, J.-W., \& Wu, X.-M. 2013, ApJL, 769, L14

Gray, D. F. 2005, The Observation and Analysis of Stellar Photospheres (Cambridge: Cambridge Univ. Press)

Hanslmeier, A., \& Dvorak, R. 1984, A\&A, 132, 203

Hatzes, A. P. 2013, AN, 334, 616

Hauschildt, P. H., Allard, F., \& Baron, E. 1999, ApJ, 512, 377

Holman, M. J., \& Murray, N. W. 2005, Sci, 307, 1288

Huang, X., Bakos, G. Á., \& Hartman, J. D. 2013, MNRAS, 429, 2001

Kipping, D. M. 2009a, MNRAS, 392, 181

Kipping, D. M. 2009b, MNRAS, 396, 1797

Kipping, D. M. 2011, MNRAS, 416, 689

Kipping, D. M., Bakos, G. Á., Buchhave, L., Nesvorný, D., \& Schmitt, A. 2012, ApJ, 750, 115

Kipping, D. M., Forgan, D., Hartman, J., et al. 2013a, ApJ, 777, 134

Kipping, D. M., Hartman, J., Buchhave, L. A., et al. 2013b, ApJ, 770, 101

Laskar, J. 1994, A\&A, 287, L9

Laskar, J. 2008, Icar, 196, 1

Léger, A., Rouan, D., Schneider, J., et al. 2009, A\&A, 506, 287

Lehmann, H., Tkachenko, A., Semaan, T., et al. 2011, A\&A, 526, A124

Lin, D. N. C., Bodenheimer, P., \& Richardson, D. C. 1996, Natur, 380, 606 
Lin, D. N. C., \& Ida, S. 1997, ApJ, 477, 781

Lissauer, J. J., Fabrycky, D. C., Ford, E. B., et al. 2011, Natur, 470, 53

Lissauer, J. J., Marcy, G. W., Rowe, J. F., et al. 2012, ApJ, 750, 112

Lithwick, Y., \& Wu, Y. 2012, ApJL, 756, L11

Lithwick, Y., Xie, J., \& Wu, Y. 2012, ApJ, 761, 122

Lovis, C., Ségransan, D., Mayor, M., et al. 2011, A\&A, 528, A112

Mandel, K., \& Agol, E. 2002, ApJL, 580, L171

Mazeh, T., Nachmani, G., Holczer, T., et al. 2013, ApJS, 208, 16

Murray, N., Hansen, B., Holman, M., \& Tremaine, S. 1998, Sci, 279, 69

Namouni, F. 2010, ApJL, 719, L145

Nesvorný, D., Kipping, D., Terrell, D., et al. 2013, ApJ, 777, 3

Ofir, A., \& Dreizler, S. 2013, A\&A, 555, A58

Petrovich, C., Malhotra, R., \& Tremaine, S. 2013, ApJ, 770, 24

Pont, F., Gilliland, R. L., Moutou, C., et al. 2007, A\&A, 476, 1347

Rasio, F. A., \& Ford, E. B. 1996, Sci, 274, 954

Rauer, H., \& Catala, C. 2011, in IAU Symp. 276, The Astrophysics of Planetary Systems: Formation, Structure, and Dynamical Evolution, ed. A. Sozzetti,

M. G. Lattanzi, \& A. P. Boss (Cambridge: Cambridge Univ. Press), 354

Raymond, S. N., Barnes, R., \& Mandell, A. M. 2008, MNRAS, 384, 663

Ricker, G. R., Latham, D. W., Vanderspek, R. K., et al. 2010, BAAS, 42, 459
Rivera, E. J., Laughlin, G., Butler, R. P., et al. 2010, ApJ, 719, 890

Sartoretti, P., \& Schneider, J. 1999, A\&AS, 134, 553

Shulyak, D., Tsymbal, V., Ryabchikova, T., Stütz, C., \& Weiss, W. W. 2004, A\&A, 428, 993

Steffen, J. H., Fabrycky, D. C., Agol, E., et al. 2013, MNRAS, 428, 1077

Steffen, J. H., Fabrycky, D. C., Ford, E. B., et al. 2012a, MNRAS, 421, 2342

Steffen, J. H., Ford, E. B., Rowe, J. F., et al. 2012b, ApJ, 756, 186

Szabó, G. M., Szatmáry, K., Divéki, Z., \& Simon, A. 2006, A\&A, 450, 395

Tenenbaum, P., Jenkins, J. M., Seader, S., et al. 2013, ApJS, 206, 5

Tremaine, S., \& Dong, S. 2012, AJ, 143, 94

Tsymbal, V. 1996, in ASP Conf. Ser. 108, M.A.S.S.: Model Atmospheres and Spectrum Synthesis, ed. S. J. Adelman, F. Kupka, \& W. W. Weiss (San Francisco, CA: ASP), 198

Tuomi, M. 2012, A\&A, 543, A52

Tuomi, M., Anglada-Escudé, G., Gerlach, E., et al. 2013, A\&A, 549, A48

Wang, S., Ji, J., \& Zhou, J.-L. 2012, ApJ, 753, 170

Ward, W. R. 1997, Icar, 126, 261

Wright, E. L., Eisenhardt, P. R. M., Mainzer, A. K., et al. 2010, AJ, 140, 1868

Wu, Y., \& Lithwick, Y. 2013, ApJ, 772, 74

Xie, J.-W. 2013, ApJS, 208, 22 